\title{
Pentetic Acid Calcium
}

National Cancer Institute

\section{Source}

National Cancer Institute. Pentetic Acid Calcium. NCI Thesaurus. Code C1710.

The calcium salt of pentetic acid, a synthetic chelating agent related to the chelating agent ethylenediaminetetraacetic acid (EDTA). Pentetic acid chelates with the metallic radioisotopic moieties of unbound, extracellular radioimmunotherapeutic agents, resulting in higher specific tumor cell binding of radioimmunotherapeutic agents; this results in improved tumor cell radiocytotoxicity and the sparing of normal cells and tissues from the radiocytotoxic effects of these agents. 